\title{
Análise multivariada de atributos do solo em sistemas convencional e orgânico de produção de maçãs
}

\author{
Carolina Riviera Duarte Maluche-Baretta ${ }^{(1)}$, Cassandro Vidal Talamini do Amarante $^{(2)}$ e Osmar Klauberg Filho(2)
}

\begin{abstract}
${ }^{(1)}$ Escola Superior de Agricultura Luiz de Queiroz, Dep. de Solos e Nutrição de Plantas, Av. Pádua Dias, no 11, Caixa Postal 9, CEP 13418-900 Piracicaba, SP. E-mail: cmaluche@esalq.usp.br ${ }^{(2)}$ Universidade do Estado de Santa Catarina, Centro de Ciências Agroveterinárias, Av. Luiz de Camões, 2090, CEP 88520-000 Lages, SC.E-mail: amarante@cav.udesc.br, a2okf@cav.udesc.br
\end{abstract}

\begin{abstract}
Resumo - Este estudo foi conduzido em pomares de maçãs nos sistemas de manejo convencional e orgânico, com o objetivo de identificar diferenças entre os dois sistemas de produção, com base em atributos microbiológicos e químicos do solo, por meio de métodos multivariados, como a análise canônica discriminante (ACD) e a análise de correlação canônica (ACC). Em ambos os pomares, foram feitas amostragens em 24 plantas distribuídas em uma grade de 45x54 m, em duas épocas, para a quantificação de teores de carbono da biomassa microbiana (CBM), carbono orgânico total do solo (COT), nitrogênio da biomassa microbiana (NBM), nitrogênio total do solo (NT), relação CBM:COT, relação NBM:NT, respiração basal $\left(C-\mathrm{CO}_{2}\right)$ e quociente metabólico $\left(q \mathrm{CO}_{2}\right)$, além da determinação de atributos químicos destes solos. A ACD identificou o CBM como o atributo microbiológico mais importante pela análise multivariada, na separação entre os pomares, seguido do $q \mathrm{CO}_{2}$ e da relação NBM:NT. Atributos microbiológicos e químicos relacionados ao carbono foram mais sensíveis às variações entre os sistemas do que os relacionados ao nitrogênio. Houve alta correlação canônica entre os atributos microbiológicos e químicos do solo nos pomares, com destaque para o CBM, entre os atributos biológicos, e para o $\mathrm{pH} \mathrm{H}_{2} \mathrm{O}$ e alumínio, entre os atributos químicos.
\end{abstract}

Termos para indexação: qualidade do solo, atividade microbiana, carbono da biomassa microbiana, nitrogênio da biomassa microbiana.

\section{Multivariate analysis of soil attributes of apple orchards under conventional and organic production systems}

\begin{abstract}
This study was carried out in apple orchards under conventional and organic production systems, aiming to identify differences between them, in terms of soil microbiological and chemical attributes, with multivariate canonical discriminant analysis (CDA) and canonical correlation analysis (CCA). Soil samples were collected under the canopies of 24 plants which were arranged in a $45 \times 54 \mathrm{~m}$ grid, in conventional and organic orchards. Samples were collected in two seasons and microbial biomass carbon (MBC), total organic carbon (TOC), microbial biomass nitrogen (MBN), total nitrogen (TN), MBC:TOC ratio, MBN:TN ratio, basal respiration $\left(\mathrm{C}-\mathrm{CO}_{2}\right)$, and metabolic quotient $\left(q \mathrm{CO}_{2}\right)$, and soil chemical attributes determined. The canonic discriminant analysis identified $\mathrm{MBC}$ as the most important microbiological attribute that discriminates orchard management systems, followed by $q \mathrm{CO}_{2}$ and $\mathrm{MBN}$ :TN rate. The microbiological and chemical attributes related to carbon were more sensitive to the orchard management systems than attributes related to nitrogen. The canonical correlation between microbiological and chemical attributes was highly significant, with a higher contribution of MBC for microbial attributes, and of $\mathrm{pH} \mathrm{H}_{2} \mathrm{O}$ and aluminium for chemical attributes.
\end{abstract}

Index terms: soil quality, microbial activity, microbial biomass carbon, microbial biomass nitrogen.

\section{Introdução}

A cultura da maçã desempenha papel importante na economia da região sul do Brasil. Verifica-se, no entanto, uma preocupação com os riscos à saúde humana e com os efeitos ambientais indesejáveis associados com o uso de produtos químicos sintéticos na produção de maçã convencional. Diante disso, a agregação de valor ao produto orgânico e a redução dos custos de produção vêm despertando o interesse dos produtores e consumidores.

O conjunto dos processos de produção da agricultura orgânica são baseados na fertilidade do solo como função direta do seu conteúdo de matéria orgânica (Ormond et al., 2002). Segundo Altieri (1999), em sistemas agroecológicos complexos, interações de caráter positivo 
entre a biota do solo promovem melhorias em sua estrutura e fertilidade, resultando em ambiente favorável a inúmeros processos biológicos (Glover et al., 2000; Reganold et al., 2000).

Processos biológicos, tais como, o aumento da colonização por fungos micorrízicos (Werner, 1997), transformação da matéria orgânica, estocagem de nutrientes (Dalal, 1998; Glover et al., 2000; Goh et al., 2000, 2001), entre outros indicativos de aumento na atividade biológica do solo, são indicativos das mudanças ocorridas no ambiente pela prática de manejo orgânico e respondem mais rapidamente às mudanças do que os atributos químicos e físicos do solo (Gunapala \& Scow, 1998; Swezey et al., 1998; Goh et al., 2000, 2001). Estudos sobre microbiologia do solo em sistemas orgânicos permitem avaliar modificações ocorridas no início do processo de conversão à prática orgânica, por meio da identificação dos atributos microbiológicos associados ao aumento da qualidade do sistema, complementando os estudos químicos e físicos.

As informações obtidas por meio desses estudos serão tão mais precisas quanto melhor o método utilizado para o tratamento estatístico dos dados. Os modelos estatísticos clássicos tornam-se menos sensíveis em sistemas biológicos, em razão das particularidades próprias de cada manejo, e não consideram o efeito conjunto de inúmeros fatores e características para promover as respostas ao manejo. A análise estatística multivariada, entretanto, permite detectar e descrever padrões estruturais, espaciais e temporais nas comunidades biológicas, e formular hipóteses baseadas nos numerosos fatores bióticos e abióticos que interferem sobre tais características (Valentin, 2000).

A análise canônica discriminante (ACD) permite identificar diferenças entre grupos e/ou tratamentos, o que resulta na compreensão das relações entre todos os atributos estudados e os grupos e/ou tratamentos (CruzCastillo et al., 1994). Por tais características, a análise multivariada torna-se eficaz em estudos microbiológicos quando se pretende identificar os atributos que servem para separar áreas de estudo, por inexistência de similaridade. Isto permite ainda a eliminação do tempo usado com atributos que expressam baixa resposta.

O objetivo deste trabalho foi identificar diferenças entre os sistemas de produção convencional e orgânico de maçãs quanto aos atributos microbiológicos e químicos do solo, por meio de modelos multivariados que utilizam a ACD e ACC.

\section{Material e Métodos}

Este estudo foi conduzido no Município de Urupema, região serrana de Santa Catarina, na altitude média de 1.425 m. O município apresenta clima mesotérmico úmido (Cfb), segundo a classificação de Köppen, com inverno e verão brandos e médias das temperaturas máximas e mínimas mensais para o período do estudo (outubro de 2002 a setembro de 2003) de $26,5^{\circ} \mathrm{C}$ e $3,3^{\circ} \mathrm{C}$, respectivamente. As chuvas são bem distribuídas durante todo o ano, com leve deficiência hídrica de dezembro a fevereiro, e maiores níveis de precipitação no período de inverno. O somatório da precipitação no período do estudo foi de $1.317 \mathrm{~mm}$.

As áreas estudadas constituíram-se de pomares de maçãs sob os sistemas convencional (PC) e orgânico (PO) de produção, com dez anos de idade. As cultivares utilizadas foram Royal Gala e Fuji, enxertadas sobre o porta-enxerto M-7, com espaçamento de $6 \mathrm{~m}$ entre linhas e $4 \mathrm{~m}$ entre plantas nas linhas de plantio. Os pomares distanciam-se aproximadamente em $1,5 \mathrm{~km}$ e estão localizados em posição similar de toposseqüência, em um terreno suavemente ondulado, com presença freqüente de afloramento de rochas. Ambos foram implantados em Cambissolos húmicos (Embrapa, 1999), com variação no teor de argila de 410 a $550 \mathrm{~g} \mathrm{~kg}^{-1}$ e elevado conteúdo de matéria orgânica (Tabela 1 ).

As áreas dos pomares eram constituídas originalmente de campos nativos, com presença de espécies como Araucaria angustifolia. No caso do pomar

Tabela 1. Caracterização química do solo, na camada de $0-10$ cm, em pomares de macieira conduzidos nos sistemas de produção convencional e orgânico, em Urupema, SC. Média de 24 repetições.

\begin{tabular}{|c|c|c|c|c|c|c|c|c|c|}
\hline \multirow[t]{2}{*}{ Pomar } & \multicolumn{2}{|c|}{$\mathrm{pH}$} & $\mathrm{P}$ & $\mathrm{K}$ & $\mathrm{Ca}$ & $\mathrm{Mg}$ & $\mathrm{Na}$ & $\mathrm{Al}$ & \multirow{2}{*}{$\begin{array}{r}\mathrm{MO}^{(3)} \\
\left(\mathrm{g} \mathrm{kg}^{-1}\right)\end{array}$} \\
\hline & $\mathrm{H}_{2} \mathrm{O}^{(1)}$ & $\mathrm{SMP}^{(2)}$ & ------(1 & -3 )------ & ------- & $--(\mathrm{cn}$ & $\left.n^{-3}\right)--$ & ----- & \\
\hline Convencional & 6,2 & 6,8 & 13,0 & 203,3 & 12,6 & 7,8 & 0,1 & 0,0 & 69,0 \\
\hline Orgânico & 5,6 & 5,8 & 8,5 & 117,3 & 9,4 & 5,4 & 0,2 & 0,1 & 77,0 \\
\hline
\end{tabular}

${ }^{(1)} \mathrm{pH}$ em água. ${ }^{(2)} \mathrm{pH}$ em solução SMP. ${ }^{(3)}$ Matéria orgânica do solo. 
convencional, a área foi utilizada para cultivo de batata, antes de sua implantação. Em 1992, os dois pomares foram implantados sob o sistema convencional de produção de maçãs; um dos pomares foi convertido ao sistema orgânico sete anos depois da implantação.

Os pomares conduzidos nos sistemas convencional e orgânico foram arranjados em filas alternadas de macieiras das cultivares Royal Gala e Fuji, com arranjo, densidade e sistema de condução das plantas similares. $\mathrm{O}$ efeito da cultivar foi desconsiderado, e foram realizadas amostragens apenas nas linhas da cultivar Royal Gala. Em cada pomar foi estabelecida uma grade de amostragem com 24 pontos. Cada ponto correspondeu a macieiras da cultivar Royal Gala distribuídas em quatro linhas de plantio, cada linha com seis plantas, com distância de $18 \mathrm{~m}$, e as plantas distanciadas $9 \mathrm{~m}$ entre si, perfazendo uma área amostrada de $2.430 \mathrm{~m}^{2}$ (45x54 m) em pomares com aproximadamente 5 ha.

Foram adicionados ao PC 500 g por planta de adubo mineral fórmula 20-10-20, $13 \mathrm{~kg}$ por planta de esterco bovino e $200 \mathrm{~g}$ por planta de fosfato natural, além de aplicações de $\mathrm{Ca}, \mathrm{Zn}$, B e P. Foram realizadas 35 aplicações de fungicidas e cinco aplicações de inseticidas. Não foi realizado controle químico de invasoras, e permitiu-se o crescimento da vegetação espontânea com controle por meio de roçadas.

O manejo do PO incluiu poda leve de inverno e raleio manual de frutos, roçada total da área em outubro, e na projeção da copa em janeiro, adubação pós-colheita utilizando-se $5 \mathrm{t} \mathrm{ha}^{-1}$ de esterco de cavalo (baseado em análise de solo), $50 \mathrm{~kg} \mathrm{ha}^{-1}$ de fosfato natural de Arad e $103 \mathrm{~kg} \mathrm{ha}^{-1}$ de sulfato de potássio (70\% desta adubação de março a abril, e 30\% em de agosto). Segundo as normas de produção orgânica, foram realizadas ainda, aplicações do composto "supermagro" (biofertilizante) e controle fitossanitário com caldas bordaleza e sulfocálcica.

Para as análises microbiológicas (biomassa microbiana e sua atividade - respiração basal) e químicas, foram coletadas amostras de solo no raio de projeção das copas das plantas selecionadas nas grades, na profundidade de $0-10 \mathrm{~cm}$. Para as análises microbiológicas, as coletas foram realizadas nos dias 10 de dezembro de 2002 (verão) e 12 de junho de 2003 (inverno), enquanto para as análises químicas, foi realizada apenas a coleta na primeira data (verão). As amostras foram acondicionadas em sacos de plástico, transportadas para o laboratório em caixas de isopor com gelo e armazenadas em geladeira a $2^{\circ} \mathrm{C}$.
As amostras coletadas foram homogeneizadas e passadas em peneiras com malhas de $2 \mathrm{~mm}$, tendo parte destas sido secada ao ar para determinação do carbono orgânico total do solo (COT), utilizado posteriormente no cálculo da matéria orgânica do solo (MO), pH em água, pH SMP e teores de $\mathrm{P}, \mathrm{K}, \mathrm{Al}, \mathrm{Na}$, $\mathrm{Ca}$ e $\mathrm{Mg}$ trocáveis, conforme método descrito em Tedesco et al. (1995). Uma subamostra foi retirada para determinação da umidade (coletas de verão e inverno),

Na determinação do $\mathrm{C}$ e $\mathrm{N}$ da biomassa microbiana (CBM e NBM) das amostras de solo, foi utilizado o método de fumigação-extração (Vance et al., 1987). Em cada ponto amostrado, foram realizadas seis repetições, sendo três amostras fumigadas e três amostras não fumigadas. Após a fumigação com clorofórmio livre de etanol $\left(\mathrm{CHCl}_{3}\right)$, o CBM foi extraído com sulfato de potássio $0,5 \mathrm{~mol} \mathrm{~L}^{-1}$ e oxidado pelo dicromato de potássio $66,7 \mathrm{mmol} \mathrm{L}^{-1}$. O teor de $\mathrm{C}$ foi determinado por titulação com sulfato ferroso amoniacal 33,3 mmol L-1 $\left(\mathrm{Fe}\left(\mathrm{NH}_{4}\right)_{2}\left(\mathrm{SO}_{4}\right)_{2} \cdot 6 \mathrm{H}_{2} \mathrm{O}\right)$, na presença de difenilamina (1\%). O NBM foi determinado utilizando-se as alíquotas extraídas pelo método da fumigação-extração para a análise do CBM. O N contido no extrato foi determinado por pré-digestão e destilação, conforme o método Kjeldahl, sendo o destilado titulado com ácido sulfúrico $25 \mathrm{mmol} \mathrm{L}^{-1}$ (Brookes et al., 1985). $\mathrm{O}$ COT e o $\mathrm{N}$ total do solo (NT) foram determinados pelos métodos de Walkley \& Black e Kjeldahl, respectivamente, conforme Tedesco et al. (1995). Para a determinação do $\mathrm{N}$ mineral $\left(\mathrm{NH}_{4}{ }^{+}, \mathrm{NO}_{3}{ }^{-}\right)$, após a coleta, as amostras foram imediatamente extraídas com $\mathrm{KCl} 1 \mathrm{M}$, sendo as alíquotas armazenadas em congelador e posteriormente destiladas com adição de $\mathrm{MgO}$ e liga Devarda, e tituladas em solução ácida (Tedesco et al., 1995).

A atividade microbiana foi avaliada pela determinação da respiração basal $\left(\mathrm{C}-\mathrm{CO}_{2}\right)$ em $100 \mathrm{~g}$ de amostras de solo, colocadas em frascos de $500 \mathrm{~mL}$ e incubadas em laboratório pelo período de 48 horas, à temperatura de $28^{\circ} \mathrm{C}$, segundo Jäggi (1976).

Com bases nos resultados de CBM, COT, NBM e NT foram calculadas as relações CBM:COT e NBM:NT, expressas como porcentagem de C e N microbiano em relação ao $\mathrm{C}$ e $\mathrm{N}$ total do solo (Anderson, 1994). Com os resultados de $\mathrm{C}-\mathrm{CO}_{2}$ e do CBM, calculou-se o quociente metabólico $\left(q \mathrm{CO}_{2}\right)$, que representa a quantidade de $\mathrm{C}-\mathrm{CO}_{2}$ liberada em determinado tempo, por unidade de C microbiano. 
Os dados foram submetidos à análise multivariada do tipo ACD, utilizando o SAS versão 6.12 (SAS Institute, 1990), para identificação dos atributos microbiológicos mais relevantes na separação das áreas estudadas dos sistemas convencional e orgânico de produção de maçãs. Como neste estudo a ACD permite apenas uma função canônica discriminante, uma segunda função discriminante foi utilizada com observações aleatórias $[\mathrm{N}(0,1)]$ para representação gráfica. A fim de esclarecer os resultados obtidos pela análise multivariada de todos os atributos, foram realizadas análises canônicas discriminantes complementares, separando-se os efeitos da contribuição de atributos microbiológicos e químicos relacionados ao $\mathrm{C}$ e ao $\mathrm{N}$ do solo. Tal procedimento foi adotado para estudar a resposta separada da dinâmica do $\mathrm{N}$ e do C, encontrada nos pomares, e a eficiência desses atributos, em razão da sensibilidade em predizer mudanças significativas entre os sistemas.

Foram realizadas ACC entre os atributos microbiológicos (CBM, C- $\mathrm{CO}_{2}, q \mathrm{CO}_{2}$ e $\mathrm{NBM}$ ) e químicos (NT, COT, $\mathrm{pH} \mathrm{H}_{2} \mathrm{O}, \mathrm{N}-\mathrm{NH}_{4}, \mathrm{~N}_{-} \mathrm{NO}_{3}$ e teores de $\mathrm{P}, \mathrm{K}, \mathrm{Na}$, Ca, $\mathrm{Mg}$ e Al trocáveis) avaliados.

\section{Resultados e Discussão}

\section{Análise canônica discriminante}

Em virtude de apenas dois tratamentos (correspondentes às áreas de PC e PO) terem sido estudados, somente uma função discriminante canônica foi utilizada, já que o número máximo de funções canônicas discriminantes é calculado pelo menor valor, de acordo com o número de grupos estudados menos um, e do número de atributos avaliados menos um (CruzCastillo et al., 1994).

O teste estatístico multivariado de Wilks’ Lambda demonstrou diferenças significativas entre as áreas estudadas $(\mathrm{p}<0,0001)$ quanto à função canônica discriminante $1\left(\mathrm{FCD}_{1}\right)$. A FCD $\mathrm{F}_{1}$ apresentou correlação canônica de 0,92, que indica elevada associação entre os atributos microbiológicos e químicos estudados, e os locais de coleta desses atributos (PC e PO).

A contribuição dos atributos microbiológicos e químicos para a separação das áreas correspondentes aos pomares convencional e orgânico é representada pelos valores de coeficiente canônico padronizado (CCP), coeficiente de correlação (r) e coeficiente da taxa de discriminação paralela (TDP) (Tabela 2).
O CCP explica o comportamento multivariado dos diferentes atributos para promover a separação entre as áreas, em resposta ao estudo de variáveis independentes, analisadas simultaneamente (Hair et al., 1987), enquanto o $r$ fornece informações univariadas da contribuição individual de cada atributo, independentemente dos demais. O melhor parâmetro para avaliação do efeito de separação gerada pelos atributos dentro das áreas estudadas é o coeficiente da TDP, que representa o produto entre CCP e r. Quanto ao coeficiente da TDP, valores positivos indicam efeito de separação entre os pomares, pois, os atributos com os maiores valores apresentam maior peso na separação entre as áreas dos pomares convencional e orgânico. Valores negativos expressam semelhanças entre os pomares quanto a esse atributo (Cruz-Castillo et al., 1994).

Quanto aos valores de CCP, o atributo CBM apresentou o maior valor positivo $(2,41)$, o que indica que a maior variabilidade encontrada entre as áreas (PC e PO) é explicada por esse atributo (Tabela 2). Ao avaliar o peso individual dos atributos, deduziu-se do valor de r que o CBM contribui com aproximadamente 47\% do total dos pesos individuais dos outros atributos (Tabela 2). Conseqüentemente, os maiores valores CCP e r encontrados para o CBM resultaram no maior valor positivo para o coeficiente da TDP na FCD 1 (Tabela 2), o que indica que a maior separação entre as áreas do PC e PO é explicada pela diferença de valores de CBM

Tabela 2. Coeficiente canônico padronizado (CCP), coeficiente de correlação (r) e coeficiente da taxa de discriminação paralela (TDP) quanto à função canônica discriminante 1 (FCD $)_{1}$, referente aos atributos biológicos e químicos de carbono da biomassa microbiana (CBM), nitrogênio da biomassa microbiana (NBM), respiração basal $\left(\mathrm{C}-\mathrm{CO}_{2}\right)$, quociente metabólico $\left(q \mathrm{CO}_{2}\right)$, nitrogênio total do solo $(\mathrm{NT})$, carbono orgânico total do solo (COT), relação carbono microbiano/ carbono total (CBM:COT) e relação nitrogênio da biomassa microbiana/nitrogênio total do solo (NBM:NT).

\begin{tabular}{lrrr}
\hline Atributos & CCP & r & TDP \\
\hline CBM & 2,41 & 0,47 & 1,14 \\
NBM & $-1,43$ & 0,19 & $-0,28$ \\
${\mathrm{C}-\mathrm{CO}_{2}}$ & 1,93 & 0,06 & 0,12 \\
$q \mathrm{CO}_{2}$ & $-2,15$ & $-0,39$ & 0,83 \\
NT & 0,32 & 0,13 & 0,04 \\
COT & $-0,83$ & 0,26 & $-0,22$ \\
Rel. CBM:COT & $-2,55$ & 0,38 & $-0,96$ \\
Rel. NBM:NT & 1,66 & 0,20 & 0,33 \\
\hline
\end{tabular}


encontrados. A análise ainda permite, por meio do coeficiente da TDP, complementar-se a separação existente entre as áreas de estudo convencional e orgânica dada pelo CBM, com a contribuição dos atributos $q \mathrm{CO}_{2}$ e relação NBM:NT, em menor grau de importância, para separar as áreas de PC e PO (Tabela 2).

Baretta et al. (2005), ao estudar o efeito do monocultivo do pinus e da queima do campo nativo em solos do Planalto Catarinense, aplicando modelos multivariados de análise de atributos microbiológicos, também identificaram o CBM como o principal atributo responsável pela separação entre áreas de estudo, por meio da análise canônica discriminante. O quociente metabólico $\left(q \mathrm{CO}_{2}\right)$ não discriminou os sistemas de produção na $\mathrm{FCD}_{1}$ (que representou $88 \%$ da variação encontrada), mas foi o atributo de maior peso na separação das áreas na segunda função discriminate $\left(\mathrm{FCD}_{2}\right)$, que respondeu por cerca de $12 \%$ da variação restante encontrada. França (2004), ao estudar sistemas de produção convencional e orgânico de citros no Estado de São Paulo, identificou o CBM e o $q \mathrm{CO}_{2}$ como atributos importantes para mensuração das mudanças ocasionadas pelo manejo. A área orgânica apresentou maiores valores de CBM e menores valores de $q \mathrm{CO}_{2}$.

Apesar de o $\mathrm{C}-\mathrm{CO}_{2}$ apresentar o segundo maior valor de CCP (Tabela 2), seu r teve menor peso de contribuição individual para a $\mathrm{FCD}_{1}$, levando ao baixo valor encontrado para o seu coeficiente da TDP. De forma semelhante ao $\mathrm{C}-\mathrm{CO}_{2}$, o NT apresentou baixo valor de r e coeficiente da TDP (Tabela 2). Observou-se por meio desses dados a menor contribuição dos atributos $\mathrm{C}-\mathrm{CO}_{2}$ e NT em aferir alterações nos sistemas de cultivo convencional e orgânico, com menor eficiência em separar os pomares estudados, pelo uso da análise multivariada. Isto se deve à maior variabilidade desses atributos nas áreas estudadas (PC e PO).

O estudo multivariado complementar para os efeitos da separação da contribuição de atributos microbiológicos e químicos relacionados ao $\mathrm{C}$ e ao $\mathrm{N}$ do solo demonstrou que a ACD para atributos microbiológicos e químicos, relacionados ao C (Tabela 3), teve diminuição da correlação canônica para a FCD 1 , comparada ao estudo conjunto dos atributos relacionados ao $\mathrm{C}$ e ao N. A correlação canônica da $\mathrm{FCD}_{1}$ para atributos relacionados ao C foi de $89,6 \%$. O teste estatístico multivariado Wilks' Lambda apresentou diferença significativa $(p<0,0001)$ entre os pomares estudados quanto a esses atributos.
O CBM apresentou os maiores valores de CCP, r e coeficiente da TDP (Tabela 3) e, ao separar atributos relacionados ao $\mathrm{C}$, na $\mathrm{FCD}_{1}$, aumentou o peso de sua participação individual na formação do eixo canônico, alcançada por meio de seu r. De todos os atributos relacionados ao C, o CBM respondeu por quase toda a separação entre pomares convencional e orgânico, portanto, foi um bom indicador das mudanças que ocorrem no ambiente, a longo prazo, pela prática de manejo (Swezey et al., 1998; Ribeiro, 2003; França, 2004; Baretta et al., 2005). Com base no coeficiente da TDP, o $q \mathrm{CO}_{2}$ contribuiu para a separação entre áreas estudadas, em menor grau (valor do coeficiente da TDP igual a 1,08) do que o CBM (Tabela 3). Esses resultados corroboram dados que indicam o $\mathrm{CBM}$ e o $q \mathrm{CO}_{2}$ como bons atributos para avaliação da qualidade do solo em sistemas agrícolas (Wardle \& Hungria, 1994; GamaRodrigues, 1999). O C-CO $\mathrm{CO}_{2}$ apresentou baixos valores do coeficiente da TDP, e, portanto, menor peso individual para a separação de áreas por meio do eixo canônico na $\mathrm{FCD}_{1}$ (Tabela 3).

Nenhuma contribuição na separação entre pomares foi dada pela relação CBM:COT e COT (Tabela 3), que apresentaram efeito supressor (valores negativos do coeficiente da TDP; -1,68 e -0,41). A relação CBM:COT é dada pela divisão direta dos valores de CBM e COT, e demonstra índice de qualidade nutricional da matéria orgânica do solo. Por ser altamente influenciada pelos valores de CBM, a utilização dessa relação poderia

Tabela 3. Coeficiente canônico padronizado (CCP), coeficiente de correlação (r) e coeficiente da taxa de discriminação paralela (TDP) quanto à função canônica discriminante 1 $\left(\mathrm{FCD}_{1}\right)$, referente aos atributos microbiológicos e químicos relacionados ao carbono - carbono da biomassa microbiana (CBM), respiração basal $\left(\mathrm{C}-\mathrm{CO}_{2}\right)$, quociente metabólico $\left(q \mathrm{CO}_{2}\right)$, carbono orgânico total (COT) e relação CBM:COT; e ao nitrogênio - nitrogênio da biomassa microbiana (NBM), nitrogênio total (NT) e relação NBM:NT do solo.

\begin{tabular}{lccr}
\hline Atributos & CCP & r & TDP \\
\hline & \multicolumn{3}{c}{ Carbono } \\
CBM & 3,38 & 0,55 & 1,85 \\
$\mathrm{C}-C O_{2}$ & 2,24 & 0,07 & 0,16 \\
$q \mathrm{CO}_{2}$ & $-2,44$ & $-0,44$ & 1,08 \\
COT & $-1,36$ & 0,30 & $-0,41$ \\
Rel. CBM:COT & $-3,86$ & 0,44 & $-1,68$ \\
\hline & \multicolumn{3}{|}{ Nitrogênio } \\
NBM & $-3,14$ & 0,67 & $-2,10$ \\
NT & 1,78 & 0,44 & 0,79 \\
Rel. NBM:NT & 3,38 & 0,68 & 2,31 \\
\hline
\end{tabular}


auxiliar na separação entre pomares. No entanto, a análise multivariada mostra que a relação CBM:COT não é um bom indicador de qualidade do solo, nos sistemas estudados de condução de pomares de macieiras.

A ACD para o grupo de atributos microbiológicos e químicos relacionados ao $\mathrm{N}$ demonstrou menor sensibilidade às variações encontradas dentro das áreas estudadas. O teste estatístico multivariado Wilks' Lambda indicou diferença $(p<0,0008)$ nesses atributos entre os pomares, porém menos expressiva do que a encontrada nos atributos relacionados ao elemento $\mathrm{C}$, e no estudo conjunto de todos os atributos estudados. A FCD apresentou correlação canônica de 56\%, o que indica menor diferença entre os pomares, conferida aos atributos relacionados ao $\mathrm{N}$, comparativamente aos atributos relacionados ao C. A relação NBM:NT apresentou os maiores valores de CCP, $\mathrm{r}$ e coeficiente da TDP (Tabela 3). O NBM se apresentou como atributo supressor (TDP $=-2,10)$ (Tabela 3).

Na Figura 1, estão indicados os coeficientes canônicos padronizados (CCP) da $\mathrm{FCD}_{1}$, em pomares de maçã conduzidos nos sistemas de produção convencional e orgânico, considerando-se todos os atributos (Figura 1 A) e, de forma individualizada, de atributos relacionados ao C (Figura 1 B) ou ao N (Figura 1 C) do solo.

A análise conjunta dos atributos microbiológicos e químicos relacionados aos elementos $\mathrm{C}$ e $\mathrm{N}$ resulta em maior sensibilidade da análise multivariada, em separar as áreas de estudo (Figura 1 A). A separação torna-se mais nítida e responde às diferenças dos conteúdos de CBM encontrados nos pomares, com os maiores valores desse atributo encontrados no PO do que no PC. Por meio da Figura $1 \mathrm{~B}$, percebe-se que o estudo multivariado de atributos microbiológicos e químicos relacionados ao $\mathrm{C}$ no solo apresenta alta sensibilidade às diferenças de manejo peculiares a cada um dos sistemas, permitindo ainda boa separação entre os pomares convencional e orgânico. No entanto, a separação entre áreas para os atributos microbiológicos e químicos relacionados ao elemento $\mathrm{N}$ apresenta menor nitidez (Figura 1 C), o que indica grande semelhança entre os pomares estudados, na consideração desses atributos.

\section{Análise de correlação canônica}

Apenas quatro correlações canônicas foram estudadas entre atributos microbiológicos e químicos, o que foi limitado pelo menor número de atributos microbiológicos. A primeira correlação canônica entre
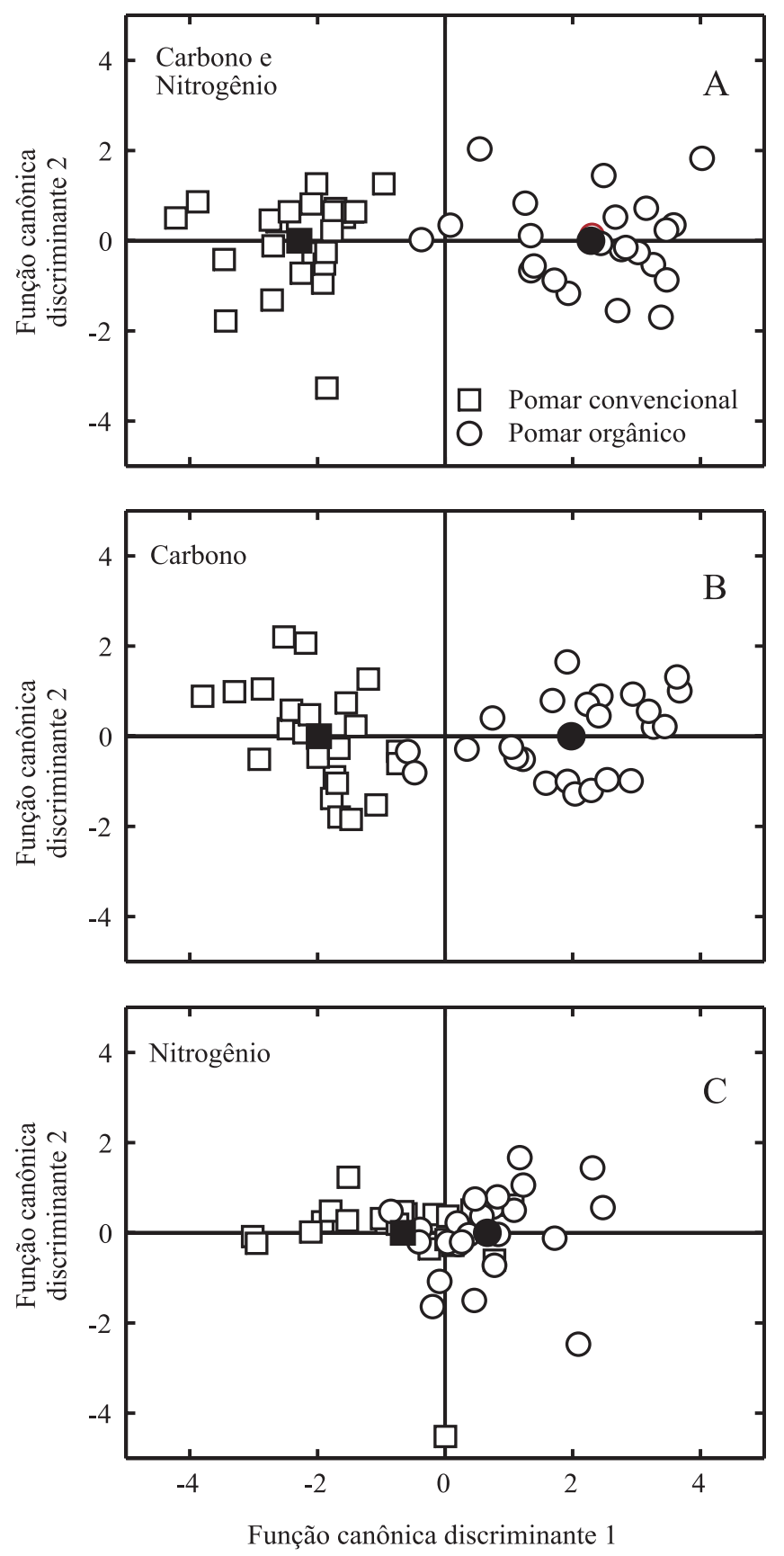

Figura 1. Coeficientes canônicos padronizados (CCP) da função canônica discriminante 1 , dos pomares de maçã, conduzidos nos sistemas de produção convencional e orgânico, considerando todos os atributos microbiológicos e químicos relacionados ao $\mathrm{C}$ e $\mathrm{N}$ do solo (A), e apenas ao C (B) ou ao N (C). Símbolo cheio representa o valor médio de CCP para cada pomar. Atributos relacionados ao carbono: CBM, COT, relação CBM:COT, respiração basal e quociente metabólico. Atributos relacionados ao nitrogênio: NBM, NT e relação NBM:NT. 
atributos microbiológicos e químicos foi de 0,90 ( $<<0,0001$ ) (Figura 2), pois 81\% da variação dos escores da primeira variável canônica dos atributos microbiológicos pôde ser explicada pelos escores da primeira variável canônica dos atributos químicos. A segunda correlação canônica foi de 0,75 ( $\mathrm{p}<0,0001)$, mas apenas $57 \%$ da variação dos escores da primeira variável canônica dos atributos microbiológicos pode ser explicada pelos escores da primeira variável canônica dos atributos químicos. A terceira e a quarta correlações canônicas não foram significativas. Desta forma, apenas a primeira correlação canônica dos dados será analisada e discutida, considerando o seu maior valor e nível de significância em relação à segunda correlação canônica.

A variável canônica biológica $1\left(\mathrm{VCB}_{1}\right)$ apresentou maiores valores de coeficientes canônicos padronizados e de correlação canônica para o CBM (1,41 e 0,83, respectivamente), seguido do $\mathrm{C}-\mathrm{CO}_{2}(-1,11$ e $-0,20$, respectivamente) (Tabela 4). Os sinais positivos de $\mathrm{CBM}$ e negativos de $\mathrm{C}-\mathrm{CO}_{2}$ indicam que áreas com elevado CBM apresentaram baixos valores de $\mathrm{C}-\mathrm{CO}_{2}$. $\mathrm{O} q \mathrm{CO}_{2}$ apresentou valor positivo para o coeficiente canônico padronizado e negativo para a correlação canônica, portanto, foi considerado um atributo supressor. O NBM apresentou valor alto de correlação canônica, mas bastante baixo de coeficiente canônico padronizado, o que indica pequena importância relativa na primeira

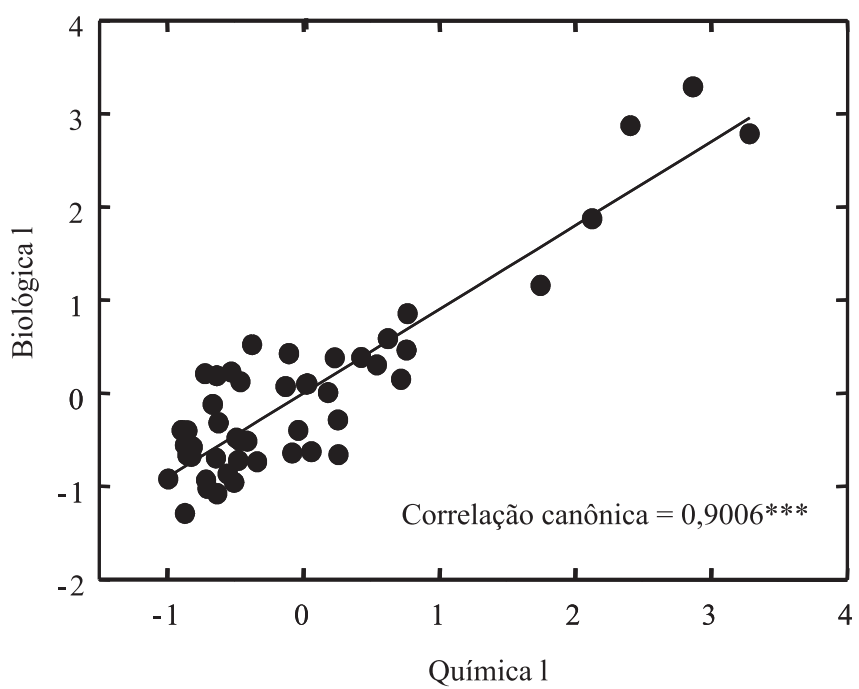

Figura 2. Análise de correlação canônica (ACC) entre atributos microbiológicos (CBM, C- $\mathrm{CO}_{2}, q \mathrm{CO}_{2}$ e NBM) e químicos (NT, COT, $\mathrm{pH} \mathrm{H}_{2} \mathrm{O}, \mathrm{N}-\mathrm{NH}_{4}, \mathrm{~N}-\mathrm{NO}_{3}$ e teores de $\mathrm{P}, \mathrm{K}, \mathrm{Na}, \mathrm{Ca}$, Mg e Al trocáveis) em pomares de maçãs conduzidos nos sistemas convencional e orgânico de produção. correlação canônica. Portanto, a maior importância relativa foi do atributo CBM, na primeira correlação com os atributos químicos estudados.

A variável canônica química $1\left(\mathrm{VCQ}_{1}\right)$ apresentou maiores valores de coeficientes canônicos padronizados e de correlação canônica para os atributos de $\mathrm{pH} \mathrm{H}_{2} \mathrm{O}$ (-0,36 e - 0,81 , respectivamente) e teores de Al trocável (0,40 e 0,76 , respectivamente) (Tabela 4$)$. Os sinais positivos de $\mathrm{Al}$ e negativos de $\mathrm{pH} \mathrm{H}_{2} \mathrm{O}$ indicam a esperada elevação nos níveis de $\mathrm{Al}$ trocável com a redução do $\mathrm{pH} \mathrm{H}_{2} \mathrm{O}$ no solo. Os atributos COT, $\mathrm{P}, \mathrm{K}$ e Ca apresentaram menor importância relativa, ao passo que os demais atributos pouco contribuíram na primeira correlação canônica com os atributos microbiológicos estudados (Tabela 4).

Houve elevada correlação canônica entre atributos microbiológicos e químicos (Figura 2), que ocorreu principalmente entre o atributo microbiológico CBM e os atributos químicos $\mathrm{pH} \mathrm{H}_{2} \mathrm{O}$ e Al. Deduziu-se destes resultados, a sensibilidade do $C B M$ às variações de $\mathrm{pH} \mathrm{H}_{2} \mathrm{O}$ e $\mathrm{Al}$ nos sistemas. Observou-se por meio da análise de correlação canônica que o CBM sofreu influência da concentração de elementos como o P, K, Ca e COT no solo. O efeito do P, na limitação ou não do crescimento da biomassa microbiana, bem como evidências da relação positiva de nutrientes como o K, Ca e o S sobre o crescimento da biomassa estão demonstrados no trabalho de Tate et al. (1991).

Tabela 4. Coeficientes canônicos homogeneizados (CCP) e correlação canônica (CC) quantos aos atributos microbiológicos das variáveis canônicas biológica $1\left(\mathrm{VCB}_{1}\right)$ e química $1\left(\mathrm{VCQ}_{1}\right)$.

\begin{tabular}{lrrr}
\hline Atributos & $\mathrm{CCP}$ & & $\mathrm{CC}$ \\
\hline $\mathrm{CBM}$ & & $\mathrm{VCB}_{1}$ & \\
$\mathrm{C}^{-\mathrm{CO}_{2}}$ & 1,41 & & 0,83 \\
$q \mathrm{CO}_{2}$ & $-1,11$ & & $-0,20$ \\
$\mathrm{NBM}$ & 0,77 & & $-0,66$ \\
& 0,16 & & 0,75 \\
Amônio & & $\mathrm{VCQ}_{1}$ & \\
Nitrato & 0,08 & & 0,17 \\
$\mathrm{NT}$ & $-0,01$ & & 0,00 \\
$\mathrm{COT}$ & 0,06 & & 0,53 \\
$\mathrm{P}$ & 0,18 & & 0,47 \\
$\mathrm{~K}$ & 0,27 & & 0,20 \\
$\mathrm{Na}$ & $-0,26$ & & $-0,45$ \\
$\mathrm{Ca}$ & $-0,06$ & & 0,22 \\
$\mathrm{Mg}$ & $-0,16$ & & $-0,74$ \\
$\mathrm{Al}$ & 0,02 & & $-0,45$ \\
$\mathrm{pH} \mathrm{H} \mathrm{H}_{2} \mathrm{O}$ & 0,40 & & 0,76 \\
& $-0,36$ & $-0,81$ \\
\hline
\end{tabular}


Os dados da correlação canônica refletem o efeito direto do aumento do $\mathrm{pH}$ pela calagem no aumento da disponibilidade de Ca no solo e seu efeito sobre a biomassa microbiana.

O estudo de correlação canônica mostrou ainda, por meio da análise de redundância, que 35\% da variação total dos atributos microbiológicos é explicada pela variação dos atributos químicos dos pomares, na primeira correlação canônica. Por sua vez, apenas $21 \%$ da variação total dos atributos químicos é explicada pela variação dos atributos microbiológicos das áreas de pomares convencional e orgânico, na primeira correlação canônica. Esses dados confirmam a sensibilidade dos atributos microbiológicos às variações químicas encontradas nos pomares estudados, e reforçam sua utilização na predição de alterações no meio antes mesmo que modificações químicas e físicas substanciais aconteçam. Suas modificações podem indicar mudanças químicas pouco perceptíveis, afetando substancialmente características microbiológicas do solo.

\section{Conclusões}

1. Métodos de análise multivariada (análise canônica discriminante e análise de correlação canônica) são importantes no estudo das alterações de atributos do solo, discriminados precocemente, nos sistemas de produção convencional e orgânico de maçãs.

2. Segundo a análise canônica discriminante, atributos relacionados ao carbono são mais sensíveis do que atributos relacionados ao nitrogênio na discriminação dos sistemas convencional e orgânico de produção em pomares de maçã.

3. O CBM é o atributo que mais contribui para discriminar os sistemas convencional e orgânico de produção de maçãs, seguido, pelo $q \mathrm{CO}_{2}$ e pela relação NBM:NT.

4. Existe alta correlação canônica entre atributos biológicos e químicos do solo nas áreas estudadas, com destaque para o CBM, entre os atributos biológicos, e para o $\mathrm{pH} \mathrm{H}_{2} \mathrm{O}$ e alumínio, entre os atributos químicos.

\section{Agradecimentos}

À Capes, Finep, Funcitec e ao CNPq, pelo apoio financeiro.

\section{Referências}

ALTIERI, M.A. The ecological role of biodiversity in agroecosystems. Agriculture, Ecosystems and Environment, v.74, p.19-31, 1999.
ANDERSON, T.H. Physiological analysis of microbial communities in soil: applications and limitations. In: RITZ, K.D.; GILLER, K.E. (Ed.). Beyond the biomass. London: British Society of Soil Science, 1994. p.67-76.

BARETTA, D.; SANTOS, J.C.P.; FIGUEIREDO, S.R.; KLAUBERG-FILHO, O. Efeito do monocultivo de pinus e da queima do campo nativo em atributos biológicos do solo no Planalto Sul Catarinense. Revista Brasileira de Ciência do Solo, v.29, p.715-729, 2005.

BROOKES, P.C.; LANDMAN, A.; PRUDEN, G.; JENKINSON, D.S. Chloroform fumigation and the release of soil nitrogen: a rapid direct extraction method to measure microbial biomass nitrogen in soil. Soil Biology and Biochemistry, v.17, p.837-842, 1985.

CRUZ-CASTILLO, J.G.; GANESHANANDAM, S.; MacKAY, B.R.; LAWES, G.S.; LAWOKO, C.R.O.; WOOLLEY, D.J. Applications of canonical discriminant analysis in horticultural research. HortiScience, v.29, p.1115-1119, 1994.

DALAL, R.C. Soil microbial biomass - what do the numbers really mean? Australian Journal of Experimental Agriculture, v.38, p.649-665, 1998.

EMBRAPA. Centro Nacional de Pesquisas de Solos (Rio de Janeiro, RJ). Sistema brasileiro de classificação de solos. Brasília: Embrapa-SPI; Rio de Janeiro: Embrapa-CNPS, 1999. 412p.

FRANÇA, S.C. Comunidade de fungos micorrízicos arbusculares nos manejos convencional e orgânico de citrus e suas interações com Phytophthora parasitica. 2004. 106p. Tese (Doutorado) - Escola Superior de Agricultura Luiz de Queiroz, Piracicaba.

GAMA-RODRIGUES, E.F. Biomassa microbiana e ciclagem de nutrientes. In: SANTOS, G.A.; CAMARGO, F.A.O. (Ed.). Fundamentos da matéria orgânica do solo: ecossistemas tropicais e subtropicais. Porto Alegre: Gênesis, 1999. p.227-244.

GLOVER, J.D.; REGANOLD, J.P.; ANDREWS, P.K. Systematic method for rating soil quality of conventional, organic, and integrated apple orchards in Washington State. Agriculture, Ecosystems and Environment, v.80, p.29-45, 2000.

GOH, K.M.; BRUCE, G.E.; DALY, M.J.; FRAMPTON, C.M.A. Sensitive indicators of soil organic matter sustainability in orchard floors of organic, conventional and integrated apple orchards in New Zealand. Biological Agriculture and Horticulture, v.17, p.197205, 2000.

GOH, K.M.; PEARSON, D.R.; DALY, M.J. Effects of apple orchard production systems on some important soil physical, chemical and biological quality parameters. Biological Agriculture and Horticulture, v.18, p.269-292, 2001.

GUNAPALA, N.; SCOW, K.M. Dynamics of soil microbial biomass and activity in conventional and organic farming systems. Soil Biology and Biochemistry, v.30, p.805-826, 1998.

HAIR, J.F.; ANDERSON, R.E.; TATHAM, R.L. Multivariate data analysis with readings. $2^{\text {nd }}$ ed. New York: Macmillan, 1987. 449p. JÄGGI, W. Die Bestimmung der $\mathrm{CO}_{2}$ Bildung ala Mass der bodenbiologischen Aktivitat. Schweizerische landwirtschaftliche Forschung, v.15, p.317-380, 1976. 
ORMOND, J.G.P.; PAULA, S.R.L. de; FAVERET FILHO, P.; ROCHA, L.T.M. da. Agricultura orgânica: quando o passado é futuro. Rio de Janeiro: BNDES, 2002. 35p.

REGANOLD, J.P.; GLOVER, J.D.; ANDREWS, P.K. Systematic method for rating soil quality of conventional, organic, and integrated apple orchards in Washington State. Agriculture, Ecosystems and Environment, v.80, p.29-45, 2000.

RIBEIRO, G.M. Características químicas, físicas e biológicas do solo em pomares de macieiras conduzidos nos sistemas orgânico e convencional de produção. 2003. 56p. Dissertação (Mestrado) - Universidade do Estado de Santa Catarina, Lages.

SAS INSTITUTE (Cary, Estados Unidos). SAS user's guide: statistics. Version 6. Cary, 1990. 2v.

SWEZEY, S.L.; WERNER, M.R.; BUCHANAN, M.; ALLISON, J. Comparison of conventional and organic apple production systems during three years of conversion to organic management in coastal California. American Journal of Alternative Agriculture, v.13, p.162-180, 1998.
TATE, K.R.; ROSS, D.J.; RAMSAY, A.J.; WHALE, K.N. Microbial biomass and bacteria in two pasture soils: an assessment of measurement procedures, temporal variations and the influence of $\mathrm{P}$ fertility status. Plant and Soil, v.132, p.233-241, 1991.

TEDESCO, M.J.; GIANELLO, C.; BISSANI, C.A.; BOHNEN, H.; VOLKWEISS, S.J. Análises de solo, plantas e outros materiais. 2.ed. Porto Alegre: UFRGS, 1995. 174p. (Boletim técnico de solos, 5).

VALENTIN, J.L. Ecologia numérica: uma introdução à análise multivariada de dados ecológicos. Rio de Janeiro: Interciência, 2000. 117p.

VANCE, E.D.; BROOKS, P.C.; JENKINSON, D.S. An extraction method for measuring soil microbial biomass C. Soil Biology and Biochemistry, v.19, p.703-707, 1987.

WARDLE, D.A.; HUNGRIA, M. A biomassa microbiana do solo e sua importância nos ecossistemas terrestres. In: ARAÚJO, R.S.; HUNGRIA, M. (Ed.). Microrganismos de importância agrícola. Brasília: Embrapa-SPI, 1994. p.193-216.

WERNER, M.R. Soil quality characteristics during conversion to organic orchard. Journal of Soil and Ecology, v.5, p.151-167, 1997.

Recebido em 11 de agosto de 2005 e aprovado em 29 de junho de 2006 